Canad. Math. Bull. Vol. 19 (2), 1976

\title{
ON A GROUP PRESENTATION DUE TO FOX
}

\author{
BY \\ C. M. CAMPBELL AND E. F. ROBERTSON
}

In 1956 R. H. Fox had occasion, while investigating fundamental groups of topological surfaces, to believe that the group $\left\langle a, b \mid a b^{2}=b^{3} a, b a^{2}=a^{3} b\right\rangle$ was trivial. Using the Todd-Coxeter coset enumeration algorithm a proof was obtained, see [3], and this algorithmic proof was used to produce an algebraic proof, see [2]. In [1] Benson and Mendelsohn, using a similar method to that of [2] showed that $\left\langle a, b \mid a b^{n}=b^{n+1} a, b a^{n}=a^{n+1} b\right\rangle$ is trivial. In this note we give a direct proof for the more general problem of describing the structure of the group $\langle a, b| a b^{n}=$ $\left.b^{\ell} a, b a^{n}=a^{\ell} b\right\rangle$.

We use $|\cdot|$ to denote the order of a group, the order of a subgroup and the modulus of an integer, the context making it clear which is intended.

THEOREM. Let $G=\left\langle a, b \mid a b^{n}=b^{\ell} a, b a^{n}=a^{\ell} b\right\rangle$. Then if

(i) $(n, \ell) \neq 1, G$ is infinite;

(ii) $(n, \ell)=1, G$ is metacyclic of order $|\ell-n|^{3}$.

Proof. We can assume without loss of generality that $n \leq \ell$.

(i) If $(n, \ell)=d \neq 1$ then adding the relations $a^{d}=b^{d}=1$ to $G$ shows that $\mathbb{Z}_{d} * \mathbb{Z}_{d}$, the free product of two copies of the cyclic group of order $d$, is a homomorphic image of $G$. Therefore $G$ is infinite.

(ii) Assume $(n, \ell)=1$. The relation $a b^{n} a^{-1}=b^{\ell}$ gives, for any $i$,

$$
a^{i} b^{n^{i}} a^{-i}=b^{\ell^{i}} \text {. }
$$

Putting $i=n$ in (1) and conjugating by $b^{-1}$ we obtain $b a^{n} b^{n^{n}} a^{-n} b^{-1}=b^{\ell n}$ and so

$$
a^{\ell} b^{n^{n}} a^{-\ell}=b^{\ell^{n}} \text {. }
$$

However (1) with $i=\ell$ is $a^{\ell} b^{n^{\ell}} a^{-\ell}=b^{\ell^{\ell}}$ and thus $b^{\ell^{n}\left(\ell^{\ell-n}-n^{\ell-n}\right)}=1$. Raising (2) to the power $\ell^{\ell-n}-n^{\ell-n}$ we obtain $b^{\left(\ell^{\ell-n}-n^{\ell-n}\right)}=1$, since $\ell$ and $n$ are coprime.

Now $\left(n, \ell^{\ell-n}-n^{\ell-n}\right)=1$ so there exist integers $\alpha, \beta$ such that $\alpha n+\beta\left(\ell^{\ell-n}-n^{\ell-n}\right)=1$. Then $G \cong\left\langle a, b \mid a b a^{-1}=b^{\alpha \ell}, b a b^{-1}=a^{\alpha \ell}, a^{\left(\ell^{\ell-n}-n^{\ell-n}\right)}=b^{\left(\ell^{\ell-n}-n^{\ell-n}\right)}=1\right\rangle$. It is easy to see that the order of $a$ and $b$ is

$$
\begin{aligned}
\left((\alpha \ell-1)^{2},\left(\ell^{\ell-n}-n^{\ell-n}\right)\right) & \\
& =\left(\alpha^{2} \ell^{2}-2 \alpha \ell+1,\left(\ell^{\ell-n}-n^{\ell-n}\right)\right) \\
& =\left(n^{2} \alpha^{2} \ell^{2}-2 n^{2} \alpha \ell+n^{2},\left(\ell^{\ell-n}-n^{\ell-n}\right)\right) \quad \text { since }\left(n^{2},\left(\ell^{\ell-n}-n^{\ell-n}\right)\right)=1 . \\
& =\left((\ell-n)^{2},\left(\ell^{\ell-n}-n^{\ell-n}\right)\right)=(\ell-n)^{2} .
\end{aligned}
$$


Now $a b a^{-1} b^{-1}=b^{\alpha \ell-1}$ so $a^{1-\alpha \ell}=b^{\alpha \ell-1}$. Raising this to the power $n$ gives $a^{n-\alpha n^{\ell}}=$ $b^{\text {ant-n}}$ showing that $a^{n-l}=b^{\ell-n}$.

Therefore $\langle a\rangle$ is normal in $G,|G|\langle a\rangle \mid=\ell-n$ and $|\langle a\rangle|=(\ell-n)^{2}$ giving the result.

COROLlaRY. The group $\left\langle a, b \mid a b^{n}=b^{n+1} a, b a^{n}=a^{n+1} b\right\rangle$ is trivial.

\section{REFERENCES}

1. C. T. Benson and N. S. Mendelsohn, A calculus for a certain class of word problems in groups, J. Combinatorial Theory 1 (1966), 202-208.

2. C. M. Campbell, Enumeration of cosets and solutions of some word problems in groups, Dissertation, McGill University, 1965.

3. H. S. M. Coxeter and W. O. J. Moser, Generators and Relations for Discrete groups (Springer, Berlin, 2nd. edition 1965).

Mathematical Institute,

UNIVERSITY OF ST. ANDREWS,

ST. ANDREWS, KY16 9SS,

SCOTLAND 